\title{
A pediatric case of primary amoebic meningoencephalitis due to Naegleria fowleri diagnosed by next-generation sequencing of cerebrospinal fluid and blood samples
}

\author{
Shiqin Huang ${ }^{1 \dagger}$, Xiu'an Liang ${ }^{1 \dagger}$, Yunli Han ${ }^{1}$, Yanyan Zhang ${ }^{1}$, Xinhui $\mathrm{Li}^{2}$ and Zhiyong Yang ${ }^{1^{*}}$ (1)
}

\begin{abstract}
Background: Primary amoebic meningoencephalitis (PAM) is a rare, acute and fatal disease of the central nervous system caused by infection with Naegleria fowleri (Heggie, in Travel Med Infect Dis 8:201-6, 2010). Presently, the majority of reported cases in the literature have been diagnosed through pathogen detection pathogens in the cerebrospinal fluid (CSF). This report highlights the first case of pediatric PAM diagnosed with amoeba infiltration within CSF and bloodstream of an 8-year-old male child, validated through meta-genomic next-generation sequencing (mNGS).
\end{abstract}

Case presentation: An 8-year-old male child was admitted to hospital following $24 \mathrm{~h}$ of fever, headache and vomiting and rapidly entered into a coma. CSF examination was consistent with typical bacterial meningitis. However, since targeted treatment for this condition proved to be futile, the patient rapidly progressed to brain death. Finally, the patient was referred to our hospital where he was confirmed with brain death. CSF and blood samples were consequently analyzed through mNGS. N. fowleri was detected in both samples, although the sequence copy number in the blood was lower than for CSF. The pathogen diagnosis was further verified by PCR and Sanger sequencing.

Conclusions: This is the first reported case of pediatric PAM found in mainland China. The results indicate that $N$. fowleri may spread outside the central nervous system through a damaged blood-brain barrier.

Keywords: Naegleria fowleri, Cerebrospinal fluid, Blood, Primary amoebic meningoencephalitis, Meta-genomics next generation sequencing

\section{Background}

Primary amoebic meningoencephalitis (PAM) is a fulminant, hemorrhagic and necrotizing meningitis [1], caused by Naegleria fowleri infection. This infection is very rare in China. The exact pathogenesis of PAM is unclear, though $N$. fowleri is often called a "brain-eating amoeba" since it causes severe encephalitis following infection,

*Correspondence: yangzhiyong@gxmu.edu.cn

† Shiqin Huang and Xiu'an Liang contributed equally to this work

${ }^{1}$ Department of Pediatrics, The First Affiliated Hospital of Guangxi

Medical University, 6 Shuangyong Road, Qingxiu District, Nanning, China

Full list of author information is available at the end of the article with a fatality rate of over 95\% [2]. PAM is an acute and progressive process, with an incubation period ranging from 2 to 15 days. Patients usually die 3-7 days after the onset of symptoms [3].

The clinical manifestations of PAM are very similar to bacterial meningitis, which makes early diagnosis difficult. This report highlights the first case of pediatric PAM in China with positive cerebrospinal fluid (CSF) and blood that was consequently confirmed through meta-genomic next-generation sequencing (mNGS). 


\section{Case presentation}

Ethical approval for this study was obtained from the Research Ethics Committee of the First Affiliated Hospital of Guangxi Medical University. The patient was an 8-year-old male admitted to hospital due to headaches, vomiting and fever during the previous $24 \mathrm{~h}$. In addition, the patient presented disturbance of consciousness for $22 \mathrm{~h}$ prior to admission. Following symptomatic treatment, vomiting was relieved although the patient still complained of fever, with the highest body temperature recorded at $39.8{ }^{\circ} \mathrm{C}$, together with persistent and severe headaches. The patient was hospitalized and consequently developed status epilepticus within $24 \mathrm{~h}$ of being admitted to hospital. The patient consequently became unconscious and required treatment with a tracheal intubation ventilator.

Table 1 Clinical test results for the cerebrospinal fluid sampled on two occasions

\begin{tabular}{llll}
\hline & June 17, 2020 & June 23, 2020 & Reference values \\
\hline $\mathrm{ICP}\left(\mathrm{mmH}_{2} \mathrm{O}\right)$ & 270 & 300 & $70-180$ \\
$\mathrm{WBC}\left(\times 10^{6} / \mathrm{L}\right)$ & 212 & 4134 & $0-5$ \\
$\begin{array}{l}\text { Percentage of } \\
\text { neutrophils }\end{array}$ & $51 \%$ & $90 \%$ & $0-2 \%$ \\
$\begin{array}{l}\text { Monocytes } \\
\mathrm{RBC}\left(\times 10^{6} / \mathrm{L}\right)\end{array}$ & $49 \%$ & $10 \%$ & Variable \\
Glucose $(\mathrm{mmol} / \mathrm{L})$ & 0.5 & $35 \%$ & 0 \\
Glucose ratio in the & 0.08 & 0.22 & $2.2-4.4$ \\
cerebrospinal fluid & & 0.04 & $>0.6$ \\
to the blood & & & \\
\begin{tabular}{l} 
Protein $(\mathrm{mg} / \mathrm{L})$ \\
\hline
\end{tabular} & 2826 & 18,740 & $<0.4$ \\
\hline
\end{tabular}

All values were measured in duplicates. ICP: intracranial pressure; WBC: Leucocyte; RBC: red blood cells (both cerebrospinal fluid examinations showed marked leukocytosis and erythrocytosis, hypoglycemia and elevated protein levels together with neutrophilic predominance of pleocytosis)
The clinical blood analyses performed consisted of the following: WBC $19.39 * 10^{9} / \mathrm{L}, \mathrm{N} \% 90.2 \%, \mathrm{Hb}$ $120 \mathrm{~g} / \mathrm{L}$, PLT $241 * 10^{9} / \mathrm{L}$, CRP $57.6 \mathrm{mg} / \mathrm{L}$. Lumbar puncture was performed, and the test results for CSF are listed in Table 1. Head CT scans identified a local density of cerebral sickle and the tentorium was slightly increased. Subarachnoid hemorrhage was not excluded (Fig. 1). The patient was given meropenem/vancomycin as an antibiotic treatment, methylprednisolone sodium succinate as an anti-inflammatory treatment, midazolam/propofol as sedative and anti-convulsion treatment, sodium valproate to control epilepsy together with active treatment to lower intracranial pressure.

Seven days later, spontaneous breathing halted and the patient fell into a deep coma with a Glasgow score of 3.0. Consequently, he was referred to the Provincial Maternal and Child Health Hospital for hospitalization. An additional lumbar puncture was performed while CSF was re-tested (Table 1). A CSF smear/culture were performed in conjunction with gamma interferon and seven viral nucleic acid tests-no abnormalities were denoted.

Head and neck magnetic resonance imaging (MRI) was performed on the 10th day post-admission. The bilateral cerebral hemispheres, basal ganglia, thalamus, brainstem and cerebellar hemispheres were found to be diffusely swollen with abnormal signals, and extensive cerebral edema was considered. Observed abnormal narrowing of the fourth ventricle, cisterna, pontine cistern, and extracerebral space, and the lower edge of the cerebellar tonsils (Fig. 2).

The patient was still treated with a broad-spectrum anti-infective treatment, based on a diagnosis of bacterial meningitis. This treatment included meropenem, vancomycin and ceftriaxone, although all proved to be ineffective.
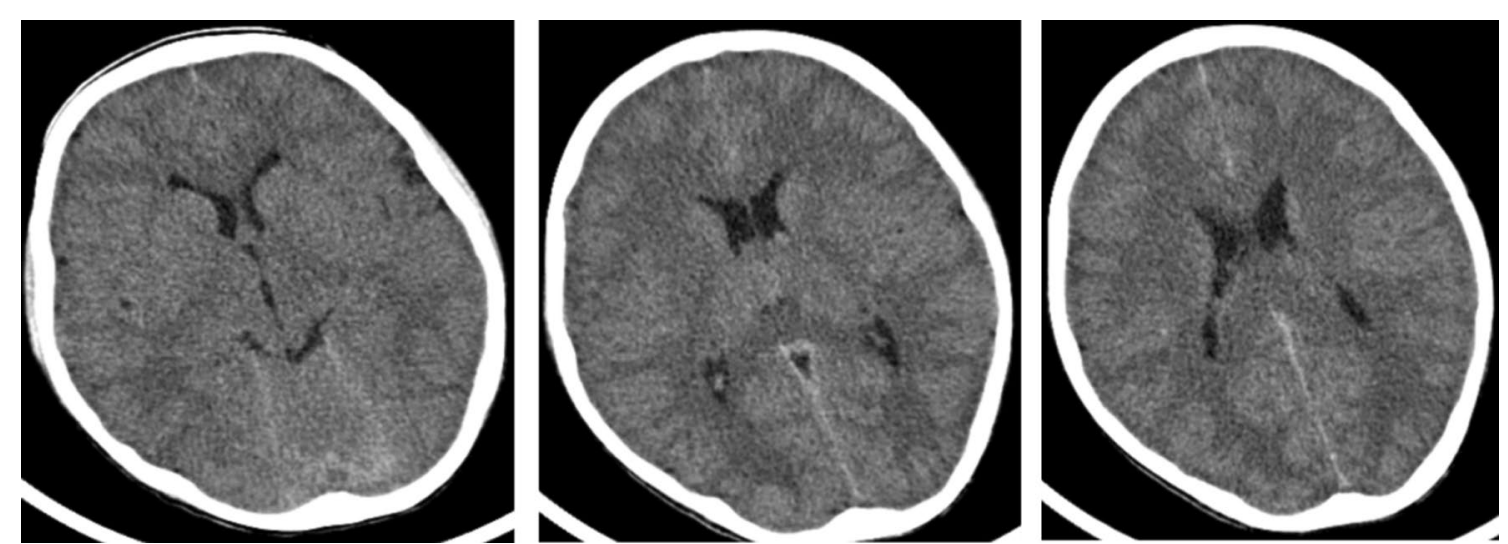

Fig. 1 Patient cranium CT scans. Local density of cerebral sickle and tentorium slightly increased, subarachnoid hemorrhage was not excluded 

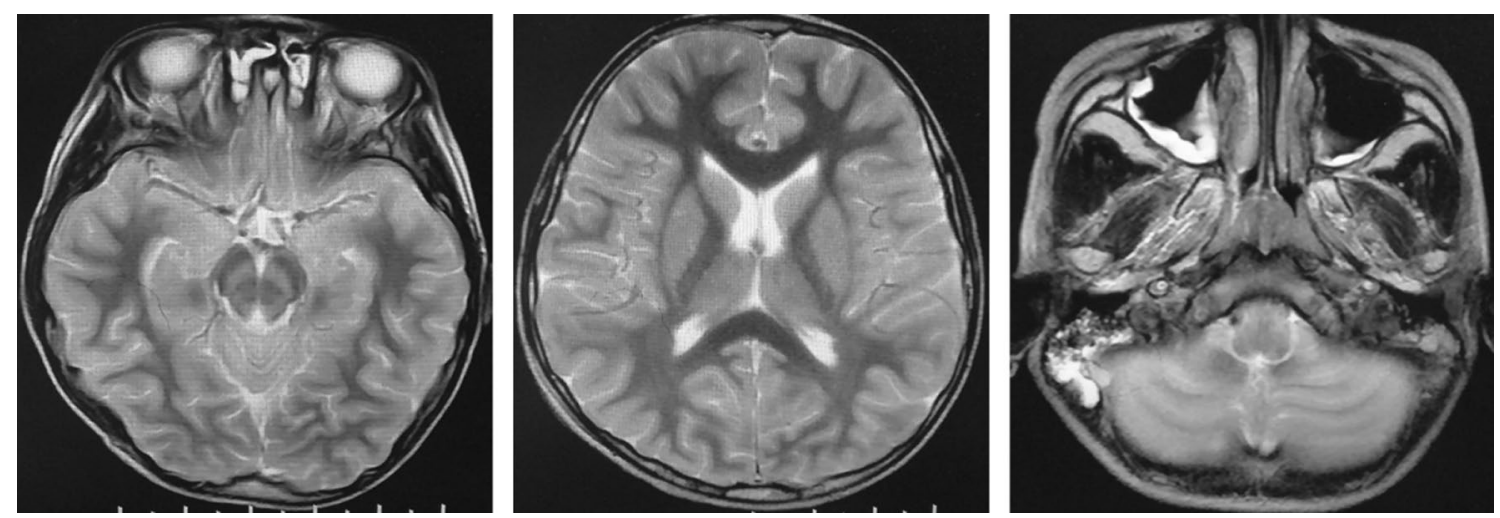

Fig. 2 Patient head/neck MRI scans of the patient. Images exhibit diffuse swelling with abnormal signals in the bilateral cerebral hemispheres, basal ganglia, thalamus, brainstem, cerebellar hemispheres and also highlights extensive cerebral edema. The fourth ventricle, ring cistern, pontine cistern and extra-cerebral space are narrow and the lower edge of the cerebellar tonsils have become pointed and moved down slightly

The patient was subsequently transferred to the First Affiliated Hospital of Guangxi Medical University, 24 days after onset of the condition and was deemed to be 'brain dead' according to the Chinese Children's Brain Death Judgment Standard [4]. A lumbar puncture was repeated, with a challenging puncture, and with only a minute amount of pale, pink, thick necrotic fluid drawn out from the syringe. The CSF, together with a blood sample were sent for mNGS [RDP-seq ${ }^{\circledR}$, Guangzhou Sagene Biotechnology Co., Ltd.]. The plasma was firstly separated from blood at $3000 \mathrm{rpm}$ for $10 \mathrm{~min}$ and consequently centrifugated at $15,000 \mathrm{rpm}$ for $5 \mathrm{~min}$. This was followed by nucleic acid extraction and purification with a nucleic acid extraction kit combined with magnetic beads [Sagene ${ }^{\mathrm{TM}}$, Guangzhou, CHINA]. A $2 \mathrm{~mL}$ CSF sample was directly centrifugated at $15,000 \mathrm{rpm}$ for $5 \mathrm{~min}$, and then were used for nucleic acid extraction and purification with nucleic acid extraction kit, as for plasma sample. The library was constructed according to the protocol for library construction Kit [Nextera $\mathrm{XT}^{\circledR}$, Illumina ${ }^{\mathrm{TM}}$, USA]. Once the libraries were mixed with equivalent amounts, high-throughput sequencing was performed on the Illumina ${ }^{\mathrm{TM}}$ Nextseq $550 \mathrm{DX}^{\circledR}$, sequencing platform (sequencing strategy: SE75), which is an FDA-approved and CE-IVD-certified sequencer. The results confirmed infection with $N$. fowleri.

A total 326 detected sequences were mapped to the $N$. fowleri genome (ASM1484362v1, genome size: $27.8 \mathrm{Mb}$ ) in CSF samples (total number of reads detected: $13,041,601)$. A total of 64 reads for the same pathogen were detected in blood samples (total number of reads detected: 15,659,034), and the analysis reliability was over $99 \%$. Concomitantly, we analyzed the distribution map of the test sequences in each sample on the genome. The read coverage against the reference genome was
$0.09 \%$ (CSF) and $0.02 \%$ (blood), respectively, with no overlap between the aligned sequence positions on the genome. PCR and Sanger sequencing were consequently employed [S-Reagent ${ }^{\circledR}$ verification system, Guangzhou Sagene Biotechnology Co. Ltd.] to further confirm pathogen infiltration:

Primer-F: CCATCATCAAAGTTAAAGGCCAC. Primer-R: GAGGAGGTTAGAATTTCATTTCGG.

The PCR reaction conditions were as follows: $3 \mathrm{~min}$ at $95^{\circ} \mathrm{C}$, followed by 40 cycles of $10 \mathrm{~s}$ denaturation at $95^{\circ} \mathrm{C}$, $30 \mathrm{~s}$ annealing at $60{ }^{\circ} \mathrm{C}, 30 \mathrm{~s}$ extension at $72{ }^{\circ} \mathrm{C}$ and $5 \mathrm{~min}$ final extension at $72{ }^{\circ} \mathrm{C}$, using a thermal cycler $\left[\mathrm{PX} 2^{\circledR}\right.$, Thermo $^{\mathrm{TM}}$, USA]. The product was $514 \mathrm{bp}$ in length. Finally, gel electrophoresis for amplified PCR products and the sequencing results of Sanger method were confirmed as positive.

\section{Discussion and conclusions}

PAM is an acute, rapid and fatal disease of the central nervous system caused by $N$. fowleri infection, prevalent mainly in children and adolescents who swim in polluted ponds or swimming pools. Approximately 440 cases of this disease has been reported worldwide, most of which have occurred within the United States, Australia and Europe [5, 6], though very few PAM cases were reported in China [7]. The clinical manifestations of PAM are similar to those of bacterial meningitis, including severe headaches, high fever, projectile vomiting, seizures, neck stiffness, changes in the level of consciousness and meningeal irritation. Within 1-12 days after the onset of initial symptoms, the condition progresses rapidly and patients often die from increased intracranial pressure and brain herniation $[8,9]$.

This case occurred in a seaside city in southern China, where the patient went swimming three days prior to 
onset of symptoms, which is consistent with the incubation period reported in the literature [3]. In this case, the patient first experienced headaches, vomiting and high fever, followed by rapid seizures, coma and finally brain death. The changes in CSF were similar to those observed in bacterial meningitis. Unenhanced head MRI highlighted diffuse cerebral edema and cerebellar tonsil hernia, which were consistent with experiences previously reported by PAM patients [10]. The condition of the patient in this case progressed rapidly and multiple antibiotic treatments were ineffective. Both the CSF and blood high-throughput sequencing results reported $N$. fowleri infiltration, further confirmed through PCR and Sanger sequencing. We suggest clinicians should ideally investigate for less common potential pathogens, in patients with bacterial meningitis who are unresponsive to first-line antibiotic treatments.

In this particular case report, $N$. fowleri was also found to be present in blood, although its sequence copy number was lower for CSF. This finding suggests that $N$. fowleri could be transmitted outside the central nervous system, which was consistent with the study of two out of the five extra-CNS tissue from autopsies examined by the CDC in the United States from 2009 to 2012. In addition to the central nervous system, $N$. fowleri was also found in the lungs, kidneys, liver, spleen and other organs of four cases reported literature [11], we speculate that $N$. fowleri can enter the bloodstream through the damaged blood-brain barrier and progress to other tissues and organs through the bloodstream, though this requires validation.

Clinically, we emphasize the early identification of less-common pathogens for this form of rare and lifethreatening brain infection, in order to achieve an early diagnosis and consequent treatment to improve patient prognosis. Furthermore, the employment of mNGS can be deemed to act as a vital diagnostic tool for rapid and accurate etiological detection of special/rare/unexpected/challenging to detect pathogens using traditional methods, such as PAM and other similar life-threatening infective conditions.

\section{Abbreviations \\ PAM: Primary amoebic meningoencephalitis; N. fowleri: Naegleria fowleri; mNGS: Meta-genomic next-generation sequencing; CSF: Cerebrospinal fluid; AmpB: Amphotericin B; CDC: Centers for Disease Control and Prevention; MRI: Magnetic resonance imaging.}

\section{Acknowledgements}

We thank Guangzhou Sagene Biotech Co. Ltd. for providing Naegleria fowlerii detection technology. The authors are also grateful to Dr. Dev Sooranna of Imperial College London for English language edits of the manuscript.

\section{Authors' contributions}

$\mathrm{SH}$ and $\mathrm{XL}$ wrote the initial draft of the manuscript, and contributed equally to this study. YH critically appraised and revised the overall content of the manuscript. $Y Z$ participated in revision of the article. $X L$ participated in the discussion and revision of the article and provided the methods of mNGS and S-Reagent test. All authors participated in the direct care and diagnosis of the patient. ZY critically reviewed the manuscript. All authors read and approved the final manuscript.

\section{Funding}

This project was supported by key R \& D Program Project of Science and Technology Bureau of Qingxiu District, Nanning City, Guangxi, China (Contract number: 2020049). This Funding section provided financial support for $\mathrm{mNGS}$ detection, but had no role in the design of the study and collection, analysis, and interpretation of data and in writing the manuscript.

\section{Availability of data and materials}

All data generated or analyzed during this study are included in this published article. The data of mNGS and PCR are upload to NCBI (mNGS Accession numbers: SRR14251192, SRR14251191; Sanger sequences Accession numbers: MW965500, MW965501).

\section{Declarations}

\section{Ethics approval and consent to participate}

Ethical approval for this study was obtained from the Research Ethics Committee of the First Affiliated Hospital of Guangxi Medical University.

\section{Consent for publish}

Written informed consent was obtained from the patient's parents for publication of this case report and the accompanying images. Copies of the written consent are available for review by the editor of this journal.

\section{Competing interests}

The authors declare that they have no competing interests.

\section{Author details}

${ }^{1}$ Department of Pediatrics, The First Affiliated Hospital of Guangxi Medical University, 6 Shuangyong Road, Qingxiu District, Nanning, China. ${ }^{2}$ Guangzhou Sagene Biotech Co., Ltd., Guangzhou, China.

Received: 1 February 2021 Accepted: 1 December 2021

Published online: 14 December 2021

\section{References}

1. Heggie TW. Swimming with death: Naegleria fowleri infections in recreational waters. Travel Med Infect Dis. 2010;8:201-6.

2. Muhammad J, Zahed M, Naveed M, et al. Naegleria fowleri: sources of infection, pathophysiology, diagnosis, and management; a review. Clin Exp Pharmacol Physiol. 2020;47:199-212.

3. Ali S, Iqbal AF, Jahan FB, et al. Fatal primary meningoencephalitis caused by Naegleria fowleri. J Coll Physicians Surg Pak. 2014;24:523-5.

4. Qian SY. Criteria and practical guidance for determination of brain death in children. Chin J Pediatr. 2019;57(5):331-5.

5. Lim YA, Nissapatorn V. Transmission of waterborne parasites in the Association of Southeast Asian Nations (ASEAN): overview and direction forward. Food Waterborne Parasitol. 2017;8:75-83.

6. Coupat-Goutaland B, Régoudis E, Besseyrias M, et al. Population structure in Naegleria fowleri as revealed by microsatellite markers. PLoS ONE. 2016;11:152434.

7. Zhang LL, Wu M, Hu BC, et al. Identification and molecular typing of Naegleria fowleri from a patient of primary amebic meningoencephalitis (PAM) in China. Int J Infect Dis. 2018;72:28-33.

8. Siddiqui $R$, Khan NA. Primary amoebic meningoencephalitis caused by Naegleria fowleri: an old enemy presenting new challenges. PLoS Negl Trop Dis. 2014;8(8):e3017.

9. Yoder JS, Eddy BA, Visvesvara GS, et al. The epidemiology of primary amoebic meningoencephalitis in the USA, 1962-2008. Epidemiol Infect. 2010;138:968-75. 
10. Capewell LG, Harris AM, Yoder JS, et al. Diagnosis, clinical course, and treatment of primary amoebic meningoencephalitis in the United States, 1937-2013. J Pediatr Infect Dis Soc. 2015;4:e68-75.

11. Roy SL, Metzger R, Chen JG, et al. Risk for transmission of Naegleria fowleri from solid organ transplantation. Am J Transplant. 2014;14:163-71.

\section{Publisher's Note}

Springer Nature remains neutral with regard to jurisdictional claims in published maps and institutional affiliations.

- fast, convenient online submission

- thorough peer review by experienced researchers in your field

- rapid publication on acceptance

- support for research data, including large and complex data types

- gold Open Access which fosters wider collaboration and increased citations

- maximum visibility for your research: over 100M website views per year

At $\mathrm{BMC}$, research is always in progress.

Learn more biomedcentral.com/submissions 